\title{
Multifractality, stickiness, and recurrence-time statistics
}

\author{
C. Vieira Abud ${ }^{1,2, *}$ and R. Egydio de Carvalho ${ }^{1, \dagger}$ \\ ${ }^{1}$ Univ Estadual Paulista-UNESP, 13506-900 Rio Claro, SP, Brazil \\ ${ }^{2}$ Universidade de São Paulo-USP, 05315-970 São Paulo, SP, Brazil
}

(Received 13 May 2013; revised manuscript received 14 September 2013; published 29 October 2013)

\begin{abstract}
We identify the fine structure of resonance islands and the stickiness in chaos through recurrence time statistics (RTS), which is based on the concept of Poincaré recurrences. The projection of recurrence time statistics onto the phase space does give relevant information on the hierarchical and microstructures of the chaotic beach around the islands of a near-integrable system, the annular billiard. These microstructures interfere in the effective transport of a particle in the phase space, which can be observed through RTS. This technique proves also to be a powerful tool to describe the homoclinic tangle of the manifolds within the chaotic sea.
\end{abstract}

DOI: 10.1103/PhysRevE.88.042922

PACS number(s): 05.45.Ac, 05.45.Pq, 45.90.+t

\section{INTRODUCTION}

The first theory of recurrences was introduced by Henri Poincare in his 1890 work [1] in which he introduced the development of recurrences in conservative systems as well as presented the idea of homoclinic tangles. Poincaré's pioneering work became more motivating with increased performance of computers, allowing numerical implementations of that concept in different branches of science. Two far-ranging reviews, which deal with Poincaré's recurrence times, can be found in Ref. [2] with a particular emphasis in synchronization of complex systems and in Ref. [3], whose focus is the concept of fractional kinetics for chaotic Hamiltonian systems.

Over the last few years one has seen a growing interest in implementing recurrence time statistics (RTS). We cite a few representative works. In Ref. [4] the authors studied a stationary flow with hexagonal symmetry, and they observed a sharp transition from normal to anomalous in the transport properties. In the anomalous scenario Poincaré's recurrence time distribution presents a power tail. They state that RTS provides a local measurement for the existence of anomalous transport. In Ref. [5] it is found that the minimum recurrence time is calculated for the lobes of an unstable manifold, and it is shown that such results are shorter than the results obtained with the usual Poincaré's recurrence time. The authors of Ref. [6] obtained an asymptotic decay law for Poincaré's recurrence as well as for the correlations by considering the standard map with divided phase space due to a critical golden curve. In Ref. [7] the author applied RTS for chaotic systems and developed scaling laws relating the mean recurrence time with the information dimension of the chaotic attractor, and he used RTS for analyzing transients and nonstationary time series. From RTS he also located bifurcations and different changes in the dynamics. A study of synchronization of chaotic systems can be found in Ref. [8], where it is shown that the dimension of Poincaré's recurrences may indicate the onset of synchronized oscillations, and in Ref. [9] the authors analyze the dependence of the mean recurrence time, and of the recurrence time statistics, on the probability density function in the interval where the recurrences are observed and in the temporal correlations of time series. For long-term

\footnotetext{
*cabud@if.usp.br

†regydio@rc.unesp.br
}

correlations, they verified the validity of the stretched exponential distribution, showing that it is restricted to the class of linear long-term correlated processes.

In Ref. [10] the authors investigated, numerically and analytically, the spectrum of Poincaré's recurrence times for area-preserving maps. They considered the limits of strong and weak coupling. In the first scenario, the spectrum typically exhibited an exponential decay, while for weak coupling they stated that for any domain of the phase space foliated by invariant tori, the asymptotic spectrum decays with $t^{-2}$. For transition regions, they proposed a combination of both laws. In that direction, the authors of Ref. [11] considered the first recurrence time in order to characterize the statistical properties of near-integrable systems. For points at the boundary of the chaotic sea and invariant tori, they posed that the statistic of the first return times is sensitive to capture the different qualitative behaviors and gives information about the relative weights played by both dynamics. In a later work [12], the authors extended this analysis to multiple return times, and they showed that for any domain involving a regular region and the chaotic sea, the statistics of visits presents a superposition of a Poissonian distribution, $\rho_{k}(\tau)=\frac{e^{-\tau}}{k !} \tau^{k}$, with a power law. They have also presented that when two or more systems are coupled, the distribution of multiple visits in domains intersecting the boundaries is a linear superposition of the individual distributions of each region. As a consequence, they showed that the real limit distributions can be masked by some finite-size effects. In a previous and formal work [13] the proofs for the exponential one and the Poisson laws are rigorously obtained. In a more recent paper [14] the authors used the first recurrence time to show that conservative systems exhibit a generalized bifurcations diagram and that all observed results agree with the results obtained with the finite time Lyapunov exponent technique.

In the present paper we continue a set of previous works [15-17], but now using RTS to characterize the thinnest structures of a nonlinear resonance. Additionally, we show that these thin structures are well recovered by plotting different components of the recurrence times. For this study we use the time-dependent, but nondissipative, annular billiard as our model. The reason to consider such a complex model is based on our experience in this system and its rich dynamics and also due to the growing interest in applying this system in some areas of physics [18-20]. 
We organize this paper as follows: in Sec. II we present RTS; in Sec. III we revisit briefly the annular billiard system; and in Secs. IV and V we present the results and conclusions, respectively.

\section{RECURRENCE TIME STATISTICS}

Initially we introduce the concept of Poincaré's recurrences for Hamiltonian systems. According to Poincaré's theorem $[21,22]$, for a confined Hamiltonian system $\Omega$, all non-nullmeasure trajectories starting in any subset $A \in \Omega$ will return many times to the neighborhood of their initial conditions. Even though the probability of a recurrence happening in a thermodynamic system is very low since the mean time for a recurrence is very large, for low-dimensional chaotic systems this mean time is measurable and can be useful.

We will develop this tool to study the vicinity of resonance islands where it is expected to have more intense stickiness for the dynamical flow. We choose our recurrence region, $A$, as large as possible involving, but not touching, the stickiness region. As we are interested in characterizing the effect of stickiness in the dynamics, we proceed as follows: We start a single initial condition in the chaotic sea (our recurrence region $A)$, and we propagate it. When it enters in the stickiness region we define the recurrence time as the time that the trajectory spends inside this region, that is, the time between the instant it entered in the stickiness region and the time that it goes back to the recurrence region. Next, we restart the time counter, but we continue propagating the same initial condition in order to store multiple recurrence times.

It is common in the literature when one calculates return times statistics to launch an ensemble of initial conditions and evaluate the times of the first return associated with each initial condition normalized by the average return time. It is expected that one collects short and long return times depending on the capture of the trajectory by the structures of stickiness. Nevertheless, for closed Hamiltonian systems, Poincaré's theorem ensures that an initial condition will repeatedly come back to the neighborhood from which it has departed. In that sense, a single initial condition will visit a certain stickiness region as many times as is desired depending only on the interval of time of observation. So one collects short and long return times in the same way as noted above.

Thus, the recurrence times will occur many times, forming a sequence of times $T_{i}=\left\{T_{1}, T_{2}, \ldots, T_{n}\right\}$ in such way that when a trajectory is trapped in a stickiness trap, $T$ corresponds to a very long time. We define a probability distribution $P(T)$ in such way that the probability of finding a recurrence time between $T_{i}$ and $T_{i}+d T_{i}$ is $P(T) d T$. The function $P(T)$ is also known as the distribution of recurrence time, and it satisfies two conditions:

$$
\begin{gathered}
\text { (1) Normalization : } \int_{0}^{\infty} P(T) d T=1 \\
\text { (2) Normalization }[23]:\langle T\rangle=\int_{0}^{\infty} T P(T) d T=\frac{1}{\mu(A)} \text {, }
\end{gathered}
$$

where $\mu(A)$ is the measure of the recurrence region. This lemma leads to finite values for the first moment and allows us to read that the probability of finding a trajectory in region
$A$ is equal to the inverse of the average recurrence time. In fact, this point is related with the ergodicity of the chaotic sea, for which it is expected that a trajectory spends, on the average, equal times in similar volumes of the chaotic phase space. In that sense, the meaning of the recurrence times is well defined from the concept of measure $\mu(A)$ of the recurrence region $A$.

For numerical experiments, it is convenient to use the distribution of the recurrence time, for times which are greater than a certain value $\tau$, through

$$
\rho(\tau)=\int_{\tau}^{\infty} P(T) d T .
$$

Expression (3) is called recurrence time statistics (RTS).

It is well established that for fully chaotic systems, RTS has an exponential decay [24],

$$
\rho(\tau) \approx e^{-\eta \tau},
$$

where $\eta$ is related with the inverse of the Kolmogorov-Sinai entropy. On the other hand, for mixed systems, for which there are stickiness regions, RTS has a power law decay,

$$
\rho(\tau) \approx \tau^{-\gamma}
$$

where $\gamma$ is called the recurrence exponent [25-27]. Conversely, if RTS obeys a power law it is sufficient to say that the analyzed region presents dynamic traps [28].

From the numeric viewpoint RTS does not depend on any ensemble of initial conditions; it is obtained from one trajectory, and this is an advantage in relation to other probability densities. So the main parameter to have a satisfactory result is the iteration number of the dynamical map, or the time in the case of continuous flux. Equation (3) is, in fact, a cumulative density of probabilities for recurrence times $T>\tau$, and numerically we calculate RTS through

$$
\rho(T)=\frac{M_{T}}{M},
$$

where $M_{T}$ is the number of recurrence with time $T>\tau$ and $M$ is the total number of recurrences along the iteration process. With this definition we start an initial condition from a chosen return region $A$, and we evolve it during a long time in order to minimize any fluctuation, which could exist if a different initial condition had been used [29]. Region $A$ is made as big as possible in order to avoid short recurrence times, which in general correspond only to random motions in the chaotic sea. We emphasize that for long times, different probability density functions (PDFs) lead to the same distribution observed for RTS because only the phase-space structures close to the stickiness region are relevant. Thus, the power-law tail of RTS can be connected with other PDFs, such as survival probabilities and decay rates [30,31]. Concerning this point, the main difference between the RTS tail and the tails of other PDFs is where the trajectories are begun. Usually survival probabilities and decay rates are calculated by an ensemble of initial condition touching the stickiness region while RTS is obtained for a single one. In this particular case a difference of $(-1)$ in the recurrence exponent is observed [25,32]. 


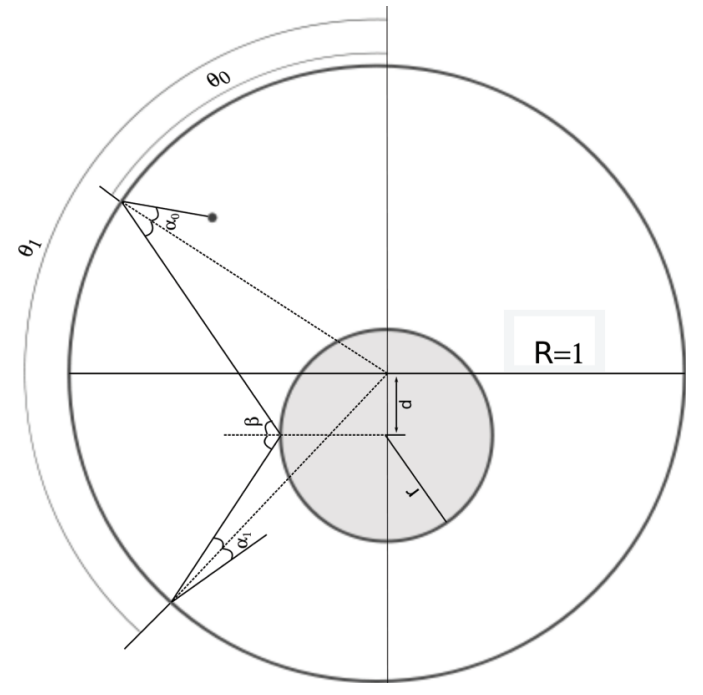

FIG. 1. A geometric scheme of a particle in the static annular billiard.

\section{THE MODEL}

In order to understand the system we are dealing with, we initially describe the dynamics of the annular billiard with static boundaries. It consists of a particle moving freely in an annular region limited by two circumscribed circumferences. They may present either the concentric or the eccentric geometries. We define the radius of the outer circle as $R=1$, the radius of the inner circle as $r$, and the eccentricity as $d$; see Fig. 1 for a pictorial example of the annular billiard. For the purpose of this work we will keep $d=0$ in all calculations; this means that the angular momentum of the particle is a constant of motion.

Inside the annulus, a particle moves along a straight line until it collides with a boundary. We will also consider only elastic collisions in this work. No dissipation is present, and the system is globally conservative for the static case and conservative in a stroboscopic fashion after introducing a periodic time-dependent perturbation. After suffering a collision the particle is specularly reflected in the sense that the incidence angle is equal to the reflection angle. From Fig. 1 we can determine the position of the particle at a collision with the external boundary through the determination of the angles $\theta$ and $\alpha$. The range of $\theta$ is $[-\pi, \pi]$ while that of $\alpha$ is $[-\pi / 2$, $\pi / 2]$. The map describing the dynamics corresponds to an application that projects the coordinates $\left(\theta_{n}, \alpha_{n}\right)$ of a collision with the outer circle, in the coordinates $\left(\theta_{n+1}, \alpha_{n+1}\right)$ of the next collision. There are also two kinds of motion, which are distinguished by the so-called tangency condition, namely,

$$
\sin \alpha_{n}-d \sin \left(\theta_{n}-\alpha_{n}\right)<r .
$$

If the combination of both, $\theta_{n}$ and $\alpha_{n}$ is such that condition (7) is not matched, then the particle does not hit the inner circle, in the current iteration, and the dynamics is described by a kind of map $M_{A}$, given by

$$
\alpha_{n+1}=\alpha_{n}, \quad \theta_{n+1}=\theta_{n}+\pi-2 \alpha_{n} .
$$

On the other hand, when condition (7) is satisfied, then between two successive collisions with the outer circle, the particle also collides with the inner circle, and the dynamics is governed by a different map given by

$$
\begin{aligned}
\alpha_{n+1} & =\arcsin \left\{r \sin (\beta)-d \sin \left(\theta_{a}\right)\right\}, \\
\theta_{n+1} & =\theta_{n}+2 \beta-\alpha_{n+1}-\alpha_{n},
\end{aligned}
$$

where

$$
\begin{aligned}
\theta_{a} & =\theta_{n+1}+\alpha_{n+1}=\theta_{n}+2 \beta-\alpha_{n}, \\
\beta & =\arcsin \left\{\frac{1}{r}\left[\sin \left(\alpha_{n}\right)-d \sin \left(\theta_{n}-\alpha_{n}\right)\right]\right\} .
\end{aligned}
$$

A convenient pair of coordinates for a plane of phase is $S=\sin (\theta)$ and $L=(\alpha / 2 \pi)$ in such way that $S=[-1,1]$ and $\mathrm{L}=[-0.5,0.5]$. We call this plane as geometric plane of phases. For the concentric, $d=0$, and static case, this plane of phases is filled by straight lines. The system is then fully integrable because, besides the angular momentum of the particle with respect to the origin of coordinate system $O$, the total energy is also preserved. This integrability can be broken through the eccentricity, for $d \neq 0$ chaotic orbits, and resonance structures can appear in the geometric plane of phases. The reader can find more details about the static annular billiard in Ref. [33].

Now we introduce a time-dependent perturbation in the boundaries in such way that both circles can breathe periodically in time. We define $\varepsilon_{R}, \varepsilon_{r}$, and $\omega$ as the amplitude of oscillation of the outer and inner circles and the ratio of frequencies of both boundaries, respectively. The particles now can suffer collisions in regions called as collision zones, which are given by $\left[1+\varepsilon_{R}, 1-\varepsilon_{R}\right]$ and $\left[r+\varepsilon_{r}, r-\varepsilon_{r}\right]$ associated with the outer and inner boundary, respectively. Figure 2 shows a schematic view of the pulsating annular billiard (PAB) with both collision zones, identified by the two rings with dotted lines. The radius of the internal boundary is described as $r_{0}$ only to emphasize that it is the radius of the nonperturbed case, or static case. This system was already studied through two distinct approaches, one called simplified model (SM) and another complete model (CM). In the CM, when a particle goes into a collision zone, it can suffer successive collisions

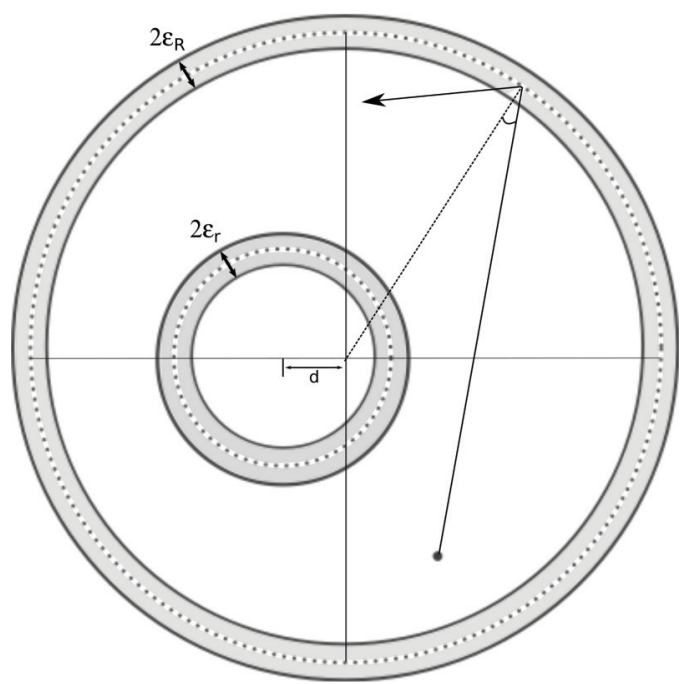

FIG. 2. A geometric scheme of a particle in the pulsating annular billiard. The collision zones are the shaded regions. 
before leaving this region. On the other hand, in the SM both boundaries are kept static, but in a collision the boundaries transfer momentum as they were pulsating, and in this case no successive collision can happen.

The algebraic details for both case can be found in Ref. [34] for the CM and Ref. [35] for the SM. The introduction of a time-dependent perturbation breaks one constant of motion, and the total energy is not preserved any longer, now it can vary; however, for the concentric case this variation is limited in a range associated with the height of the chaotic sea of a second plane of phases, called energy plane of phases. This plane is defined by the coordinates $\left(-v_{n}, \varphi\right)$ where $v_{n}$ is the normal component of the velocity of the particle and $\varphi$ is the oscillation phase of the external circle. Contrarily, for the eccentric case the particle energy can increase "unlimitedly," and the phenomenon of Fermi acceleration can be observed $[34,35]$. Nevertheless, as already mentioned above, we will develop our studies about Poincaré's recurrence using only the concentric geometry. Figure 3 shows the energy phase plane for the concentric PAB.

\section{RESULTS}

Even though, for $\delta=0$, the PAB presents spanning curves in the energy phase space $\left(-v_{n}, \varphi\right)$ there is a wide region of mixed dynamics with chaos and rich resonance structures. These resonance structures may constitute, in different ways, appropriated regions to observe stickiness and multifractality, which are characterized from the different decay laws obtained with the RTS technique. The multifractality of the main island [inside the blue box of Fig. 3(a)] is responsible for trapping trajectories during very long time. We will look at the multifractal island of Fig. 3(b) in order to evaluate RTS and the ranges of times associated with the different zooms of Fig. 3. Let us consider three zooms of the blue rectangle marked in Fig. 3(a). Figure 3(b) corresponds to the archipelago inside the rectangle of Fig. 3(a). Figure 3(c) is an enlargement of the island on the far right of Fig. 3(b). And Fig. 3(d) is a zoom of the island at the top of Fig. 3(c). We can observe that the island configurations are quite different, and this fact, associated with the multigeneration islands, which means islands of different scales, shows that this region has a multifractal structure.

The stickiness region which we are going to consider in the present study is marked in blue in Fig. 3(a) in such way that an initial condition is started in the connected chaotic sea, which is our recurrence region, outside the blue box. Note that proceeding in this way, our recurrence region $A$ will be defined as the whole accessible space outside the blue square in Fig. 3(a). The blue square we call our region of study. The main purpose of this work is to investigate if the different periods of the recurrence times carry thorough information about the deepest scales of stickiness. We iterate the initial condition up $10^{11}$, but we initialize the time counter only when the trajectory enters the stickiness region [blue box in Fig. 3(a) and emphasized in Fig. 3(b)], and we store the recurrence times every time the particle leaves this region. At this moment we reinitialize the time counter until the next entry into the stickiness region, when the time counter is again reinitialized, and this process is repeated until we exhaust the total number of iterations. The different recurrence times will be repeated
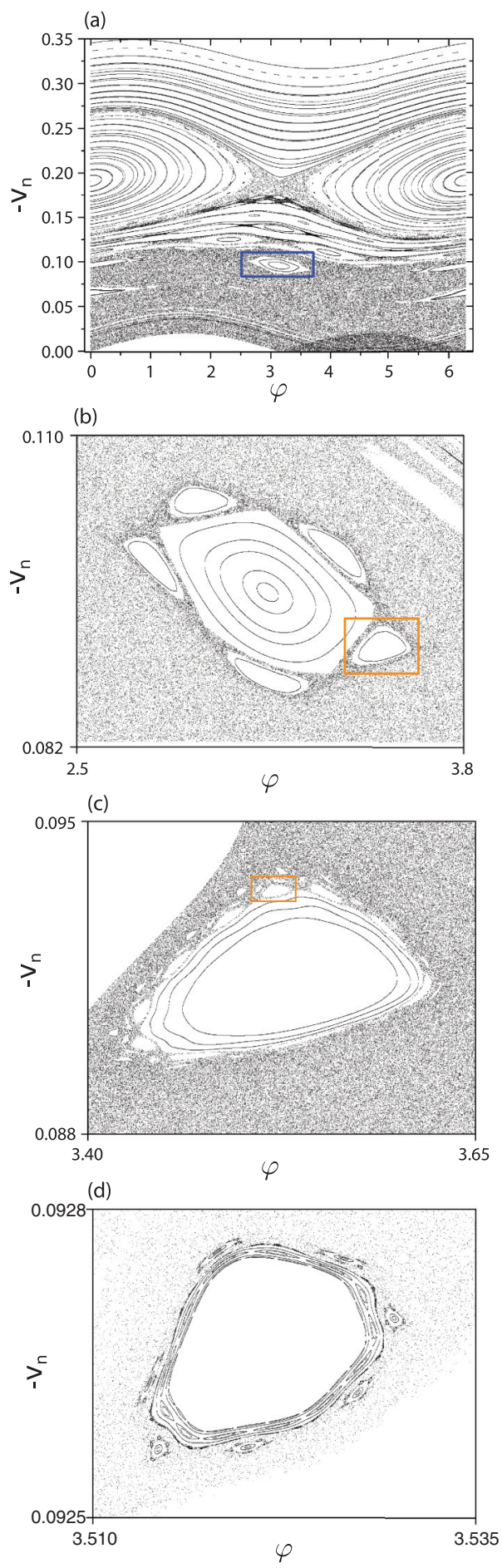

FIG. 3. (Color online) Plots of $-V_{n} \times \varphi$ for the concentric pulsating annular billiard with $\varepsilon_{R}=\varepsilon_{r}=0.01$ and $r=0.4$. (a) $\mathrm{A}$ typical plot of the system with invariant tori, chaos, and resonance structures. (b)-(d) Successive zooming of the archipelago seen in the blue rectangle of (a): (c) zoom of the orange box in (b); (d) zoom of the orange box in (c).

many times. So we construct the plot of RTS as a function of $\tau$, as shown in Fig. 4 .

Figure 4 shows that for short times, $\tau<20$, the decay is approximately exponential, as previously noted, and this behavior corresponds to random motions into the chaotic sea 


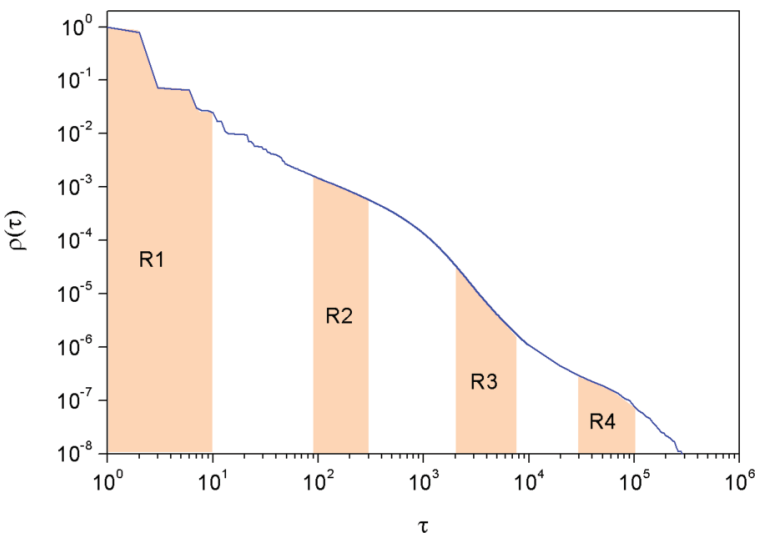

FIG. 4. (Color online) RTS for the island structures observed in Fig. 3(b). The intervals of time $R_{1}, R_{2}, R_{3}$, and $R_{4}$ have been chosen to study the different kind of time decay. $R_{1}$ [1:10]; $R_{2}$ [100:300]; $R_{3}\left[2 \times 10^{3}: 8 \times 10^{3}\right] ; R_{4}\left[3 \times 10^{4}: 1 \times 10^{5}\right]$.

outside of any trap. Figure 5(a) corroborates this. On the other hand, for intermediate and long recurrence time, the decay is best represented by power laws. If the zoomed islands of Fig. 1 had a self-similarity, it would be reasonable to expect that a unique power law would be enough to represent RTS. However, as this is not the case, then RTS must be represented by different power laws, each one for a different range of time. These times are classified by intervals identified by $R_{1}, R_{2}, R_{3}$, and $R_{4}$. We point out that the choice of these four intervals was made to understand the origin of different trapping regimes that can be characterized by different decay laws of RTS in Fig. 4. The time corresponding to each interval was chosen without any explicit reason, but we stress that different intervals of times related to the same decay should generate the same result.

Now we apply the RTS technique in order to uncover the microstructures inside the island of Fig. 3(b) as well as to get the homoclinic walk of the manifolds. The procedure consists in dividing the phase space presented in Fig. 3(b) into a grid of $10^{3} \times 10^{3}$ points to construct a density of trajectories for each interval $R_{k}$ previously defined. The plot of the density is obtained from the evolution of the same initial condition after $10^{11}$ iterations and separating the trajectories that return in each time $T \in R_{k}$. We projected four density plots on the phase space. They are shown in Figs. 5(a)-5(d), from which we can observe a very high quality in their similarity with Fig. 3

In fact, they are a decomposition of Fig. 3(b) in components of recurrence times. Figure 5(a) corresponds to the region $R_{1}$, which is identified by short recurrence time associated with random walk into the chaotic sea. Figure 5(b) corresponds to the region $R_{2}$ characterized by intermediate recurrence times. This plot shows fine contours that are similar to the manifolds of the hyperbolic fixed points present in the energy phase space of the annular billiard. We call these contours projected manifolds, and they form the homoclinic tangle separating the near-integrable islands and the sea of chaos. Figure 5(c) corresponds to the region $R_{3}$ whose recurrence times are reasonably long, and the plot shows second generation islands immersed into a layer of chaos. In this regime the trajectories spend a lot of time around the manifolds, reported in region $R_{2}$, and next they go toward the second generation of islands, where they remain practically trapped. Observing the plots (a)
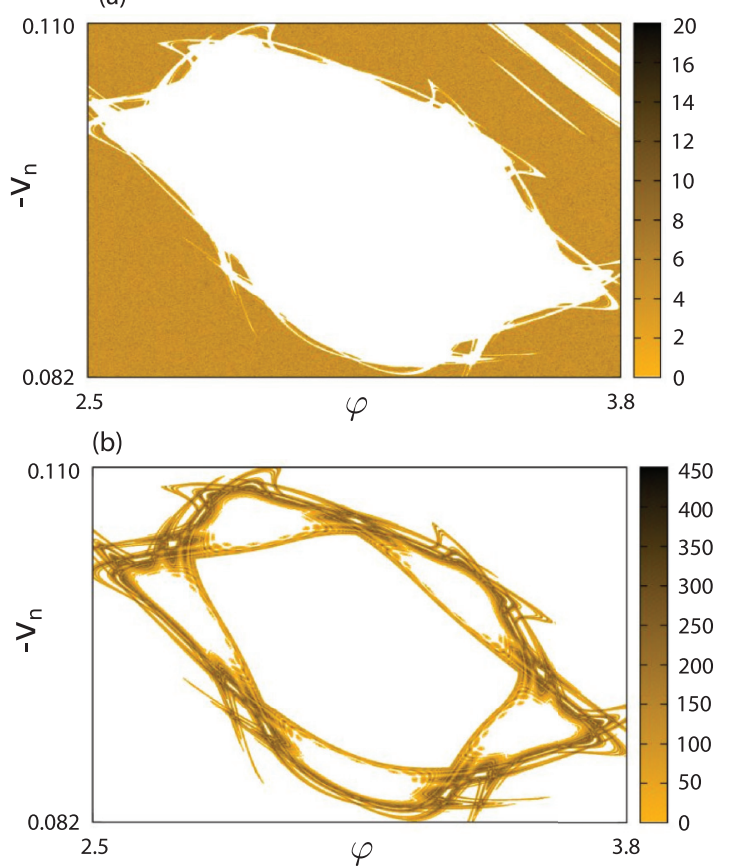

(c)

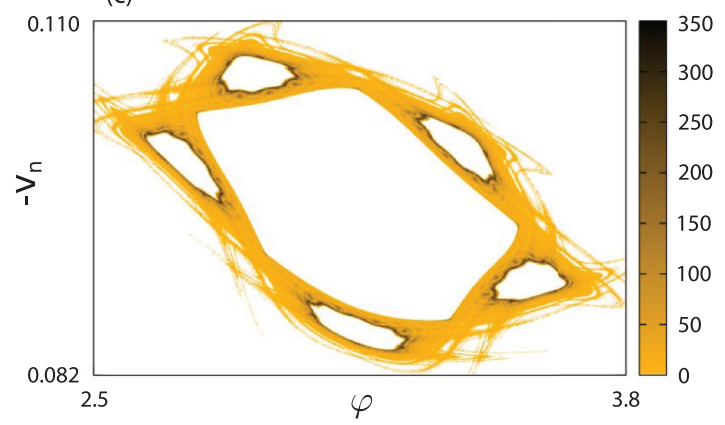

(d)

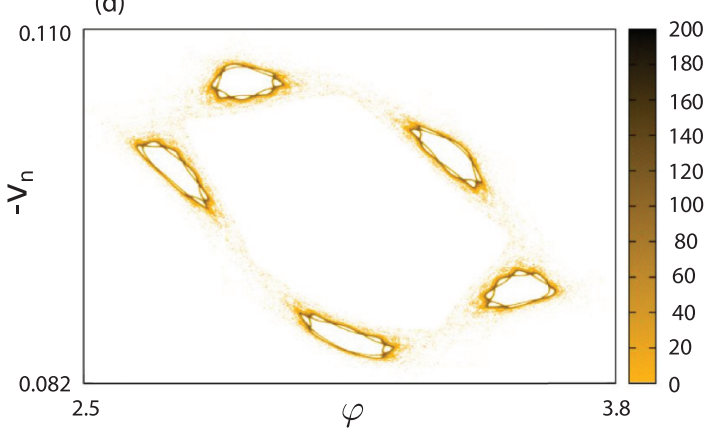

FIG. 5. (Color online) Projection of RTS on the phase space. We see the density of points, associated with Fig. 3(b), for trajectories with the recurrence times selected in Fig. $4, R_{1}$ (a), $R_{2}$ (b), $R_{3}$ (c), and $R_{4}(\mathrm{~d})$.

of Figs. 5(b) and 5(c), it seems reasonable to accept that a trajectory is first trapped by the manifolds and then by the second generation of islands. A similar result has been reported in Ref. [36], whose trapping, due to the manifolds, the author identifies as stickiness in chaos. However, in his approach the corresponding recurrence time is not identified. Finally, Fig. 5(d) corresponds to the region $R_{4}$ from where it is possible to observe that the trajectory has attained an inner region of 
the resonance structure. This regime is associated with very long recurrence times, and because of that, the finest structures and their essence are visited and uncovered. Conversely, these finest islands are responsible for the long time trapping. A remarkable aspect of this regime is that when a trajectory attains the interval $R_{4}$, it does not spend too much time visiting other intervals and rapidly converges to the inner structures of scales.

\section{CONCLUDING REMARKS}

We apply the technique of recurrence time statistics in order to identify and characterize the multifractality and stickiness of a nonlinear mixed system.

The technique of RTS has been applied in the annular billiard model in order to study and identify the finest resonance structures of the phase space that trap the particles for different time scales. The RTS presented different behaviors, and we observed that there is not only a decay law, which shows that the dynamics comprises different layers of stickiness, each one trapping the particle in a proper way. This point reinforces that the dynamics presents different resonance structures for different scales and highlights its multifractality. Besides being a powerful tool to study low-dimensional Hamiltonian chaotic systems, RTS shows an interesting feature, namely, it splits the different motions and geometries which are present in the phase space.
In the literature, it is well established that strong chaotic systems present an exponential behavior for the recurrence times distribution and that for near-integrable systems this distribution presents a polynomial form. The annular billiard model, which we are considering, is such a complex system, due to the different possibilities of movements, so we expect intermediate patterns between these limits. Our numerical evidences suggest that there are different polynomial decays for RTS due to the stickiness caused by the multiscale resonance structures, and we point out that our results can be applied to any other low-dimensional dynamical system.

We would like to pose a final comment about the richness of the annular billiard: it presents a peculiarity when we keep constant the caustic of radius $(r+\delta)$ and simultaneously increase the "parameter of perturbation $\delta$ "; the scattering inner disk tends to a point, and the dynamics tends to be regularized. However, a unique nonperiodic trajectory fills densely the phase space as the system was chaotic. This point will be subject of new investigation in the light of the recurrence times.

\section{ACKNOWLEDGMENT}

The authors thank the Brazilian scientific agency FAPESP for financial support through grants 2007/57992-4 and 2010/20276-2.
[1] H. Poincaré, Acta Mathematica 13, 1 (1890), http://link.springer. com/journal/11511/13/1/page/1

[2] N. Marwan, M. C. Romano, M. Thiel, and J. Kurths, Phys. Rep. 438, 237 (2007).

[3] G. M. Zaslavsky, Phys. Rep. 371, 461 (2002).

[4] G. M. Zaslavsky and M. K. Tippett, Phys. Rev. Lett. 67, 3251 (1991).

[5] G. Contopoulos and C. Polymilis, Celest. Mech. Dyn. Astron. 63, 189 (1995).

[6] B. V. Chirikov and D. L. Shepelyansky, Phys. Rev. Lett. 82, 528 (1999).

[7] J. B. Gao, Phys. Rev. Lett. 83, 3178 (1999).

[8] V. S. Afraimovich, W.-W. Lin, and N. F. Rulkov, Int. J. Bifurcation Chaos 10, 2323 (2000).

[9] E. G. Altmann and H. Kantz, Phys. Rev. E 71, 056106 (2005).

[10] N. Buric, A. Rampioni, G. Turchetti, and S. Vaienti, J. Phys. A 36, L209 (2003).

[11] H. Hu, L. Rossi, G. Turchetti, and S. Vaienti, Chaos 14, 160 (2004).

[12] N. Haydn, E. Lunedei, L. Rossi, G. Turchetti, and S. Vaienti, Chaos 15, 033109 (2005).

[13] M. Hirata, B. Saussol, and S. Vaienti, Commun. Math. Phys. 206, 33 (1999).

[14] C. Manchein and M. W. Beims, Phys. Lett. A 377, 789 (2013).

[15] R. Egydio de Carvalho, C. Vieira Abud, and F. Caetano Souza, Phys. Rev. E 77, 036204 (2008).

[16] R. Egydio de Carvalho and C. Vieira Abud, Chaos Solitons Fractals 44, 569 (2011).

[17] C. V. Abud and R. E. de Carvalho, Phys. Rev. E 84, 036204 (2011).

[18] M. Hentschel and K. Richter, Phys. Rev. E 66, 056207 (2002).
[19] E. G. Altmann, Phys. Rev. A 79, 013830 (2009).

[20] A. Kamor, F. Mauger, C. Chandre, and Turgay Uzer, Phys. Rev. E 85, 016204 (2012).

[21] E. Ott, Chaos in Dynamical Systems (Cambridge University Press, New York, 2002).

[22] I. P. Cornfield, S. V. Fomin, and Y. G. Sinai, Ergodic Theory (Springer, New York, 1992).

[23] M. Kac, Probability and Related Topics in Physical Science (Interscience, New York, 1959).

[24] G. M. Zaslavsky, Chaos in Dynamical Systems (Harwood, New York, 1985).

[25] J. D. Meiss, Chaos 7, 139 (1997).

[26] B. V. Chirikov and D. L. Shepelyansky, Physica D 13, 395 (1984).

[27] C. F. F. Karney, Physica D 8, 360 (1983).

[28] G. M. Zaslavsky, M. Edelman, and B. A. Niyazov, Chaos 7, 159 (1997).

[29] G. Cristadoro and R. Ketzmerick, Phys. Rev. Lett. 100, 184101 (2008).

[30] E. G. Altmann, Ph.D. thesis, Universität Wuppertal, 2007.

[31] E. G. Altmann, J. S. E. Portela, and T. Tél, Rev. Mod. Phys. 85, 869 (2013).

[32] A. S. Pikovsky, J. Phys. A: Math. Gen. 25, L477 (1992).

[33] O. Bohigas, D. Boosé, R. Egydio de Carvalho, and V. Marvulle, Nucl. Phys. A 560, 197 (1993).

[34] R. E. de Carvalho, F. C. Souza, and E. D. Leonel, Phys. Rev. E 73, 066229 (2006).

[35] R. Egydio de Carvalho, F. Caetano de Souza, and E. D. Leonel, J. Phys. A 39, 3561 (2006).

[36] G. Contopoulos and M. Harsoula, Int. J. Biff. Chaos 18, 2929 (2008). 